\title{
Dietary Micronutrients and Risk of Chronic Kidney Disease: A Cohort Study with 12 Year Follow-Up
}

\author{
Juyeon Lee ${ }^{1,2,3}\left(\mathbb{D}\right.$, Kook-Hwan $\mathrm{Oh}^{4}$ and Sue-Kyung Park $1,3,5, * \mathbb{D}$ \\ 1 Department of Preventive Medicine, College of Medicine, Seoul National University, 103 Daehakro, \\ Jongnogu, Seoul 03080, Korea; juyeon87@snu.ac.kr \\ 2 Department of Biomedical Science, College of Medicine, Seoul National University, 103 Daehakro, Jongnogu, \\ Seoul 03080, Korea \\ 3 Department Cancer Institution, Seoul National University, 103 Daehakro, Jongnogu, Seoul 03080, Korea \\ 4 Division of Nephrology, Department of Internal Medicine, Seoul National University Hospital, 103 Daehakro, \\ Jongnogu, Seoul 03080, Korea; khoh@snu.ac.kr \\ 5 Integrated Major in Innovative Medical Science, College of Medicine, Seoul National University, \\ 103 Daehakro, Jongnogu, Seoul 03080, Korea \\ * Correspondence: suepark@snu.ac.kr; Tel.: +82-2-740-8338
}

check for updates

Citation: Lee, J.; Oh, K.-H.; Park, S.-K. Dietary Micronutrients and Risk of Chronic Kidney Disease: A Cohort Study with 12 Year Follow-Up. Nutrients 2021, 13, 1517. https:// doi.org/10.3390/nu13051517

Received: 12 March 2021

Accepted: 27 April 2021

Published: 30 April 2021

Publisher's Note: MDPI stays neutral with regard to jurisdictional claims in published maps and institutional affiliations.

Copyright: (c) 2021 by the authors. Licensee MDPI, Basel, Switzerland. This article is an open access article distributed under the terms and conditions of the Creative Commons Attribution (CC BY) license (https:/ / creativecommons.org/licenses/by/ $4.0 /)$.

\begin{abstract}
We investigated the association between dietary micronutrient intakes and the risk of chronic kidney disease (CKD) in the Ansan-Ansung study of the Korean Genome and Epidemiologic Study (KoGES), a population-based prospective cohort study. Of 9079 cohort participants with a baseline estimate glomerular filtration rate (eGFR) $\geq 60 \mathrm{~mL} / \mathrm{min} / 1.73 \mathrm{~m}^{2}$ and a urine albumin to creatinine ratio (UACR) $<300 \mathrm{mg} / \mathrm{g}$ and who were not diagnosed with CKD, we ascertained 1392 new CKD cases over 12 year follow-up periods. The risk of CKD according to dietary micronutrient intakes was presented using hazard ratios (HRs) and 95\% confidence intervals (95\% CIs) in a full multivariable Cox proportional hazard models, adjusted for multiple micronutrients and important clinico-epidemiological risk factors. Low dietary intakes of phosphorus ( $<400 \mathrm{mg} /$ day), vitamin B2 ( $<0.7 \mathrm{mg} /$ day) and high dietary intake of vitamin B6 ( $\geq 1.6 \mathrm{mg} /$ day) and C ( $\geq 100 \mathrm{mg} /$ day) were associated with an increased risk of CKD stage $3 \mathrm{~B}$ and over, compared with the intake at recommended levels $(\mathrm{HR}=6.78[95 \% \mathrm{CI}=2.18-21.11] ; \mathrm{HR}=2.90[95 \% \mathrm{CI}=1.01-8.33] ; \mathrm{HR}=2.71$ [95\%CI $=1.26-5.81] ; \mathrm{HR}=1.83[95 \% \mathrm{CI}=1.00-3.33]$, respectively). In the restricted population, excluding new CKD cases defined within 2 years, an additional association with low folate levels $(<100 \mu \mathrm{g} /$ day $)$ in higher risk of CKD stage 3B and over was observed $(\mathrm{HR}=6.72[95 \% \mathrm{CI}=1.40-32.16])$. None of the micronutrients showed a significant association with the risk of developing CKD stage 3A. Adequate intake of micronutrients may lower the risk of CKD stage $3 \mathrm{~B}$ and over, suggesting that dietary guidelines are needed in the general population to prevent CKD.
\end{abstract}

Keywords: micronutrient; minerals intake; vitamins intake; chronic kidney disease

\section{Introduction}

Chronic kidney disease (CKD) is defined either as at least 3 months of a reduced estimated glomerular filtration rate (eGFR) $<60 \mathrm{~mL} / \mathrm{min} / 1.73^{2}$ or kidney damage, or as the presence of persistent albuminuria [1]; it is considered a significant public health problem with an increasing prevalence. According to different countries, including the United States (U.S.), CKD affects 8-16\% of adults worldwide [2]. In the Korean population, the prevalence of CKD is $8.2 \%$ [3].

Early diagnosis of CKD is important for reducing mortality, improving treatment outcomes, and managing complications such as cardiovascular disease (CVD) [4]. Potential risk factors for CKD include older age, diabetes mellitus, hypertension, obesity, alcohol consumption, smoking, and poor dietary habits. Dietary intake, as a modifiable risk factor, plays an important role in CKD development $[5,6]$. It has been reported in three studies 
that higher intake of certain antioxidant vitamins or healthy diet (e.g., Mediterranean diet) may improve kidney function or reduce CKD [7-9]. Despite epidemiologic evidence, the associations of dietary minerals and vitamins with the development of CKD have not been universally defined, and the results for each micronutrient have been inconsistent because most studies either used the cross-sectional study design or focused on patients on dialysis $[7,10]$. In particular, it is not clear how much to eat to prevent CKD, and what is the appropriate intake, which is defined as the minimum risk for developing CKD.

In the present study, we investigated the overall and subgroup risk of CKD stage 3A and stage $3 \mathrm{~B}$ and over according to dietary micronutrient intake using the 12 year followup data of the Ansan-Ansung cohort from the Korea Genome and Epidemiologic Study (KoGES). The subgroup was classified into diabetic CKD, hypertensive CKD, and other CKD by underlying causes (subjects who had diabetes at the time of baseline measurement and subsequently developed CKD were defined as diabetic CKD; subjects who had hypertension (HTN) at the time of baseline measurement and subsequently developed CKD were defined as hypertensive CKD).

\section{Materials and Methods}

\subsection{Study Population}

The Ansan-Ansung study, a prospective population-based cohort study, is part of the KoGES. Further details of the design and methodologies of the Ansan-Ansung study (Ansan: representing the urban community, Ansung: representing the rural community) and the KoGES have been presented elsewhere [11]. This research is an ongoing prospective cohort study involving biennial follow-ups. At baseline, 10,038 participants (aged 40-69 years), including 5020 from Ansan and 5018 from Ansung, were recruited between 2001 and 2002 through the cluster sampling method. Subjects were followed up until the 6th regular follow-up survey conducted between 2013 and 2014. At each visit, informed written consent was obtained from all subjects. Among the 10,030 subjects, we excluded those with a low eGFR $\left(<60 \mathrm{~mL} / \mathrm{min} / 1.73 \mathrm{~m}^{2}\right)$ at the cohort enrollment, as calculated using the Chronic Kidney Disease Epidemiology Collaboration (CKD-EPI) formula, those with a urine albumin to creatinine ratio $\geq 300 \mathrm{mg} / \mathrm{g}$, those with a known history of kidney disease $(n=485)$, those without information on serum creatinine levels $(n=2)$, those who did not complete a food frequency questionnaire (FFQ) $(n=307)$, and those with a history of cardiovascular disease $(n=157)$ (Figure 1$)$. The final analysis was therefore conducted on 9079 participants, of whom 1392 developed CKD $\left(e G F R<60 \mathrm{~mL} / \mathrm{min} / 1.73^{2}\right)$ and $7687 \mathrm{did}$ not (eGFR $\left.\geq 60 \mathrm{~mL} / \mathrm{min} / 1.73^{2}\right)$. In addition, we performed sensitivity analyses restricted to a eGFR $<60 \mathrm{~mL} / \mathrm{min} / 1.73^{2}$ and CKD cases diagnosed within 2 years after study entry (8901 participants, of whom 1115 developed CKD and 7786 did not develop CKD).

This study was conducted according to the guidelines established by the Declaration of Helsinki. Written informed consent was obtained from all subjects, and this study was approved by the National Biobank of Korea and the Centers for Disease Control and Prevention, Republic of Korea. The study protocols were approved by the institutional review board (IRB) of Seoul National University Hospital. 


\section{Ansan and Ansung Cohort within the KoGES}

$(n=10,038)$

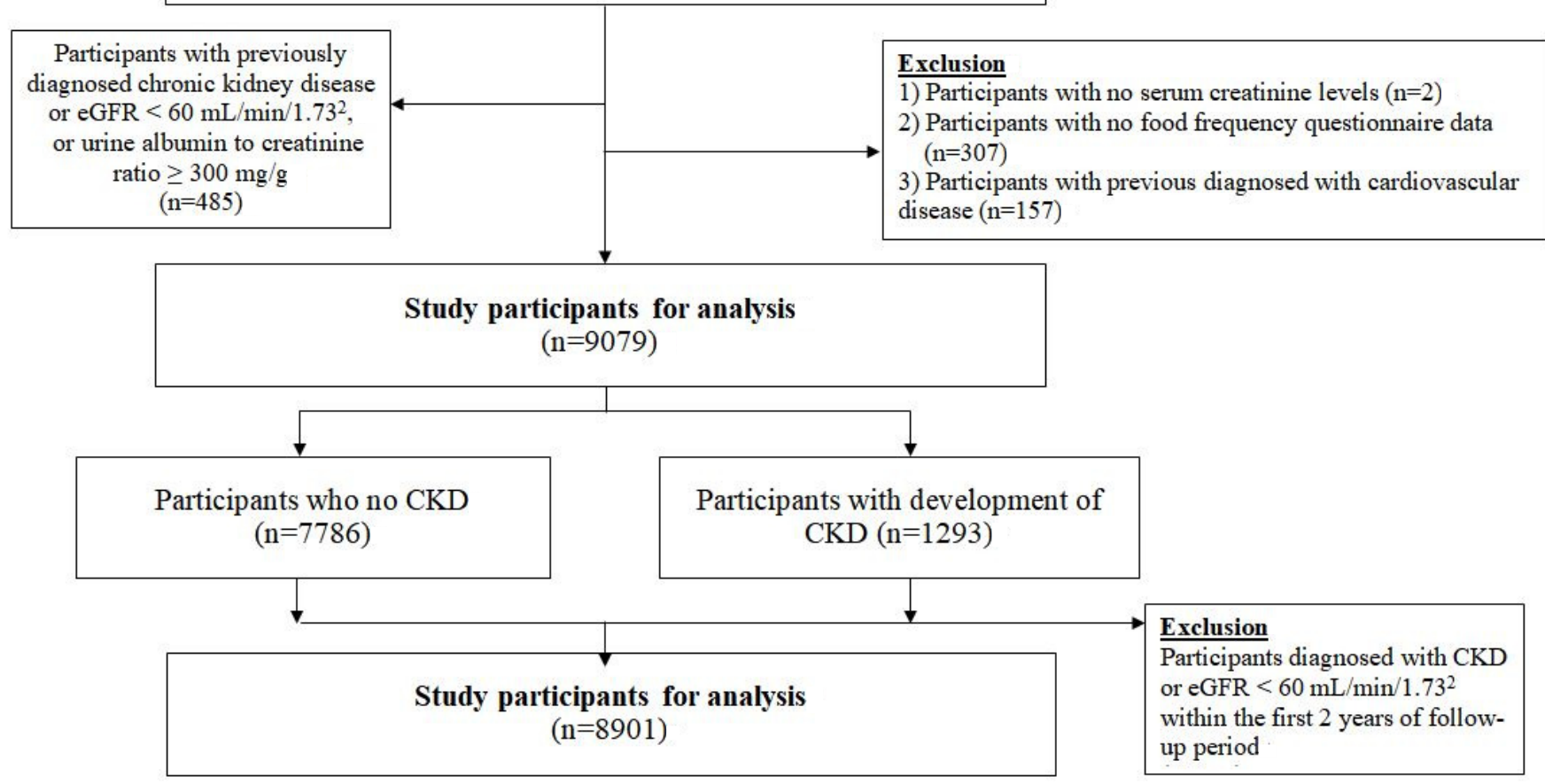

Figure 1. Study subjects to assess the association between dietary minerals and vitamins with development of chronic kidney disease, the Ansan-Ansung cohort of the Korean Genome and Epidemiologic study (KoGES). KoGES, Korean Genome and Epidemiologic Study; eGFR, estimated glomerular filtration rate; CKD, chronic kidney disease.

\subsection{Ascertainment of New CKD Cases over 12 Year Follow-Up}

CKD was defined as a 'eGFR $<60 \mathrm{~mL} / \mathrm{min} / 1.73 \mathrm{~m}^{2}$ ', in compliance with the National Kidney Foundation Kidney Disease Outcomes Quality Initiative (NKF KDOQI) clinical practice guideline [12]. A new case of CKD was defined as a eGFR decline to $<60 \mathrm{~mL} / \mathrm{min} / 1.73 \mathrm{~m}^{2}$ over a follow-up period ranging from 2 to up to 12 years among cohort members with an eGFR greater than $60 \mathrm{~mL} / \mathrm{min} / 1.73 \mathrm{~m}^{2}$ at the time of baseline. The eGFR was calculated using the CKD-EPI equation [13]. The CKD-EPI equation is shown as Equation (1).

$$
\mathrm{eGFR}=141 \times \min \left(\frac{S c r}{k}\right)^{\alpha} \times \max \left(\frac{S c r}{k}\right)^{-1.209} \times 0.993^{\text {Age }} \times 1.018[\text { if female }]
$$

$S c r=$ serum creatinine $(\mathrm{mg} / \mathrm{dL}) ; k=0.7$ if female; 0.9 if male; $\alpha=-0.329$ if female; -0.411 if male.

$\min =$ the minimum of $S c r / k$ or 1 ; $\max =$ the maximum of $S c r / k$ or 1.

Of the 9079 cohort population, the number of new cases of CKD stage 3A was 1239, of which 1140 were completed in stage $3 \mathrm{~A}$ over 12 year follow-up, and 99 were deteriorated with a eGFR from stage $3 \mathrm{~A}$ to stage $3 \mathrm{~B}, 4$, or 5 . There were 153 new patients ascertained as 'CKD stage 3B or higher', of which 99 were deteriorated from CKD stage $3 \mathrm{~A}$ as previously mentioned, and the remaining 54 were new cases who were directly ascertained as stages $3 \mathrm{~B}, 4$, or 5 without being ascertained as stage $3 \mathrm{~A}$ during biennial follow-up. Since biennially repeated measurements/follow-up was conducted in this cohort study, the 54 patients can be defined as patients who rapidly deteriorated during follow-up.

The patients ascertained as CKD stage 3A were considered as early CKD patients without any eGFR deterioration and some of them might be transient or temporary CKDs due to biennial follow-up setting of community-based cohort study. However, since 
patients ascertained as 'stage 3B or higher' were those who worsened from stage 3A during follow-up or had experienced rapid eGFR deterioration during biennial followup, these may be considered as 'definite CKD cases' on the setting of community-based cohort study. Moreover, since the risk of mortality was different between two patient group of CKD stage 3A and 3B and over [14,15], two patient groups were defined as the group with different clinical characteristics. Thus, based on this consideration, we classified CKD into three groups: non-CKD, defined as a eGFR $\geq 60 \mathrm{~mL} / \mathrm{min} / 1.73 \mathrm{~m}^{2}$; CKD stage $3 \mathrm{~A}, 45 \mathrm{~mL} / \mathrm{min} / 1.73 \mathrm{~m}^{2}<\mathrm{eGFR}<60 \mathrm{~mL} / \mathrm{min} / 1.73 \mathrm{~m}^{2}$; and stage $3 \mathrm{~B}$ and over, a eGFR $<45 \mathrm{~mL} / \mathrm{min} / 1.73 \mathrm{~m}^{2}$.

\subsection{Clinical and Laboratory Measurements}

Questionnaires surveys, clinical investigations, and physical examinations were performed during the baseline and follow-up assessments. The subjects were questioned by well-trained interviewers regarding their sociodemographic status, lifestyle (smoking, alcohol consumption, and physical activity), anthropometric measurements, and personal and family medical history. All samples were immediately sent to the Korean National Biobank; the reliability of each biomarker analysis has been previously published [10]. Blood and urine samples were collected in a serum separator tube (SST), and a two-ethylene ediaminetetra acetic acid (EDTA) tube, along with a $10 \mathrm{~mL}$ midstream urine sample. Both serum and plasma were prepared and aliquoted, and 100-800 $\mu \mathrm{g}$ of blood DNA and 6-10 vials (300-500 $\mu \mathrm{L}$ per vial) were also prepared. For each tube, a two-dimensional barcode was used as a label. Serum creatinine was assayed using the Jaffe method with a HITACHI Automatic Analyzer 7600 (Hitachi, Tokyo, Japan) and ADVIA 1650 Auto Analyzer (Siemens, Washington, DC, USA) [16].

\subsection{Dietary Assessment}

The daily nutrient intakes were assessed using a validated, semi-quantitative FFQ developed for the KoGES [16]. All subjects were asked to estimate their average serving of 106 food items and their frequency of consumption. Dietary intakes per day were calculated by combining the serving frequency and portion per unit for each food item with the average amount per serving. We divided the dietary intake of 6 minerals (calcium, phosphorus, sodium, potassium, iron, and zinc) and 10 vitamins (vitamin $\mathrm{A}$, retinol, vitamin B1, vitamin B2, niacin, vitamin B6, folate, vitamin C, carotene, and vitamin E) into recommended values and used them for further analyses. The recommended dietary allowance (RDA), adequate intake (AI), or National Kidney Foundation guideline values of each mineral and vitamin for individuals aged 51-70 years were designated as the reference category. The reference values of each mineral were as follows: calcium ( $\geq 600 \mathrm{mg} /$ day), phosphorus (700-1200 mg/day), sodium (2000-2999 mg/day), potassium ( $\geq 3400 \mathrm{mg} /$ day), iron (7-10 mg/day), and zinc (8-11 mg/day) (Tables 1 and 2); those of each vitamin were as follows: vitamin A ( $\geq 700 \mathrm{R} . \mathrm{E} /$ day), retinol ( $\geq 100 \mu \mathrm{g} /$ day), carotene ( $\geq 3400 \mu \mathrm{g} /$ day), vitamin $\mathrm{E}(\geq 11 \mathrm{mg} /$ day), vitamin B1 (0.9-1.2 mg/day), vitamin B2 (0.9-1.2 mg/day), niacin (14-19 mg/day), vitamin B9 ( $\geq 400 \mu \mathrm{g} /$ day), and vitamin C (75-100 mg/day) (Supplementary Table S1).

\subsection{Statistical Analyses}

To compare the general characteristics of the CKD $\left(e G F R<60 \mathrm{~mL} / \mathrm{min} / 1.73 \mathrm{~m}^{2}\right)$ and non-CKD (eGFR $\geq 60 \mathrm{~mL} / \mathrm{min} / 1.73 \mathrm{~m}^{2}$ ) groups, we conducted an independent t-test for continuous variables and the chi-square test for categorical variables. In order to overcome the reverse causation in the association between dietary factors and CKD incidence to some extent, a 'restricted population' group was additionally formed after excluding newly defined CKD cases within 2 years.

We examined the associations of dietary minerals and vitamins with CKD stage $3 \mathrm{~A}$ and CKD stage $3 \mathrm{~B}$ and over using the Cox proportional hazard regression models in the entire population and in the restricted population, respectively. First, to analyze the association 
with a single micronutrient on the risk of CKD, a Cox regression model was constructed by controlling the following 10 clinico-epidemiological factors, which were statistically significant in each univariable analysis: baseline age, baseline eGFR, sex, body mass index (BMI), physical activity, cigarette smoking, alcohol consumption, total cholesterol levels, the urine albumin to creatinine ratio, and uric acid in urine (Supplementary Table S2). Additionally, two factors, such as total calories and protein intake were additionally included in the multivariable model for a single micronutrient because higher nutrient levels were due to higher intake in diet and protein intake was a dietary factor that is preferentially restricted in CKD patients. We also performed stratified analyses by diabetes mellitus (DM), and hypertension (HTN) origins. DM was defined as the presence of a history of diabetes and fasting blood glucose $\geq 126 \mathrm{mg} / \mathrm{mL}$ at baseline. HTN was defined as a patient on antihypertensive medication, or the presence of a history of hypertension, systolic blood pressure $\geq 140 \mathrm{~mm} / \mathrm{Hg}$, and diastolic blood pressure $\geq 90 \mathrm{~mm} / \mathrm{Hg}$.

In order to observe the effect of one micronutrient on the risk of CKD development under adjustment for multiple micronutrients and other risk factors, we constructed a clinico-nutritional model which was a full multivariable composite model, composed of two models, a dietary nutritional model and a clinico-epidemiological model. Each dietary nutritional model and clinico-epidemiological model was constructed by backward Cox regression models. We assessed the collinearity between independent variables using the variance inflation factor (VIF) and the Pearson's correlation coefficient. The starting model of the dietary nutritional model included 6 minerals, vitamins $E$ and vitamin $C$, retinol among the vitamin $\mathrm{A}$ and its precursors, and vitamin B2, folate, and vitamin B6, which were significant in the single-vitamin analysis among the vitamin B complex. By using the backward Cox model, zinc, potassium, vitamin E were deleted. Although total energy and protein intake were not statistically significant in univariable analysis, both were included in the multivariable nutritional model because higher nutrient levels were due to higher intake in diet and protein intake is a dietary factor that is preferentially restricted in CKD patients. The starting model of the clinico-epidemiological model was constructed with 10 clinico-epidemiological factors used for single-micronutrient analysis and the stratification variables DM and HTN. By using the backward Cox model, alcohol consumption, total cholesterol levels in blood, the urine albumin to creatinine ratio, and uric acid in urine were deleted. Thus, eight variables (age, baseline eGFR, sex, BMI, physical activity, cigarette smoking, DM, and HTN) in the clinico-epidemiological model and eleven nutritional variables (calcium, phosphorus, sodium, iron, retinol, vitamin B2, folate, vitamin B6, vitamin C, total calories and protein intake) in the dietary nutritional model were finally selected. Additionally, the clinico-nutritional model was composed of 19 variables.

In addition, we evaluated the discriminatory accuracy of the dietary nutritional model, the clinico-epidemiological model and the clinico-nutritional model (a composite model of both models) in predicting CKD risk using Harrell's $C$ index. All statistical analyses were conducted using SAS version 9.4 (SAS Institute Inc., Cary, NC, USA).

\section{Results}

\subsection{General Characteristics}

Compared with the non-CKD group, the CKD group was more likely to be older; female; of higher BMI; have a lower education level (below middle school); have lower protein/fat intake, serum hemoglobin, hematocrit, HDL cholesterol, ALT, total protein, albumin, urine creatinine, uric acid, sodium, and PH levels; and have higher serum total cholesterol, triglycerides, fasting blood sugar (FBS), glycated hemoglobin (HbA1C), highsensitivity C-reactive protein (hsCRP), blood urea nitrogen (BUN), calcium, and potassium levels (Supplementary Table S2). 


\subsection{Associations between Single-Mineral/Vitamin Intake and the Risk of Developing CKD}

The associations with single-micronutrient intake on the risk of CKD stage $3 \mathrm{~A}$ or stage $3 \mathrm{~B}$ and over are shown in Table 1 . When the mineral intake was divided into categories, the lowest and highest intake levels were associated with increased risk of CKD stage $3 \mathrm{~B}$ and over compared with the reference levels, which included the RDA, AI, or National Kidney Foundation guideline values for calcium $(<200 \mathrm{mg} /$ day, HR $=2.0,95 \%$ CI: $1.01-3.63)$, phosphorus ( $<400 \mathrm{mg} /$ day, $\mathrm{HR}=6.1,95 \%$ CI: 3.22-11.58), iron ( $<7 \mathrm{mg} /$ day, $\mathrm{HR}=1.9,95 \%$ CI: $1.22-3.10 ; \geq 13.3 \mathrm{mg} /$ day, $\mathrm{HR}=2.1,95 \% \mathrm{CI}: 1.06-4.34)$, and zinc $(5 \mathrm{mg} /$ day, $\mathrm{HR}=2.5$, 95\% CI: 1.23-5.13) (Table 1).

Table 1. Single-mineral intake for $\mathrm{CKD}^{1}$ risk in the entire cohort population (the Ansan-Ansung KoGES) with 12 year follow-up $(n=9079)$.

\begin{tabular}{|c|c|c|c|c|c|c|c|c|c|}
\hline & \multirow{2}{*}{$\begin{array}{l}\text { Person- } \\
\text { Years }\end{array}$} & \multicolumn{2}{|c|}{$\begin{array}{l}\text { CKD Stage } 3 \mathrm{~A}^{1} \\
\quad(n=1140)\end{array}$} & \multirow{2}{*}{$\begin{array}{l}\text { Person- } \\
\text { Years }\end{array}$} & \multicolumn{2}{|c|}{$\begin{array}{l}\text { CKD Stage 3B and over }{ }^{1} \\
\qquad(n=153)\end{array}$} & \multirow{2}{*}{$\begin{array}{l}\text { Person- } \\
\text { Years }\end{array}$} & \multicolumn{2}{|c|}{$\begin{array}{c}\text { CKD } \\
(n=1293)\end{array}$} \\
\hline & & $\begin{array}{c}\text { Cases } \\
n\end{array}$ & $\begin{array}{c}\text { HR } \\
(95 \% \text { CI })^{2}\end{array}$ & & $\begin{array}{c}\text { Cases } \\
n\end{array}$ & $\begin{array}{c}\text { HR } \\
(95 \% \mathrm{CI})^{2}\end{array}$ & & $\begin{array}{c}\text { Cases } \\
n\end{array}$ & $\begin{array}{c}\text { HR } \\
(95 \% \text { CI })^{2}\end{array}$ \\
\hline \multicolumn{10}{|c|}{ Calcium $(\mathrm{mg})^{3}$} \\
\hline$<200$ & 6176 & 124 & $1.0(0.80-1.34)$ & 5440 & 30 & $2.0(1.01-3.62)$ & 6322 & 154 & $1.1(0.87-1.40)$ \\
\hline $200-400$ & 27,123 & 465 & $1.1(0.93-1.36)$ & 24,181 & 52 & $1.0(0.59-1.72)$ & 27,394 & 517 & $1.1(0.92-1.32)$ \\
\hline $400-600$ & 22,004 & 295 & $1.0(0.80-1.14)$ & 20,158 & 37 & $1.0(0.61-1.61)$ & 22,207 & 332 & $1.0(0.81-1.13)$ \\
\hline$\geq 600$ & 19,102 & 256 & 1.0 & 17,462 & 34 & 1.0 & 19,293 & 290 & 1.0 \\
\hline \multicolumn{10}{|c|}{ Phosphorus (mg) ${ }^{3}$} \\
\hline$<400$ & 857 & 16 & $0.9(0.52-1.41)$ & 816 & 14 & $6.1(3.22-11.58)$ & 920 & 30 & $1.3(0.90-1.90)$ \\
\hline $400-700$ & 12,934 & 249 & $1.0(0.84-1.18)$ & 11,346 & 39 & $1.7(1.04-2.65)$ & 13,150 & 288 & $1.0(0.88-1.21)$ \\
\hline $700-1200$ & 40,450 & 617 & 1.0 & 36,646 & 64 & 1.0 & 40,789 & 681 & 1.0 \\
\hline$\geq 1200$ & 20,165 & 258 & $0.8(0.70-1.01)$ & 2151 & 36 & $0.9(0.53-1.50)$ & 20,356 & 294 & $0.9(0.72-1.01)$ \\
\hline \multicolumn{10}{|c|}{ Sodium $(\mathrm{mg})^{3}$} \\
\hline$<2000$ & 16,601 & 297 & $1.0(0.89-1.22)$ & 14,682 & 40 & $1.2(0.72-1.84)$ & 16,808 & 337 & $1.0(0.90-1.22)$ \\
\hline 2000-2999 & 21,936 & 332 & 1.0 & 19,797 & 34 & 1.0 & 22,099 & 366 & 1.0 \\
\hline 3000-3999 & 17,540 & 239 & $0.9(0.78-1.09)$ & 15,973 & 33 & $1.1(0.69-1.84)$ & 17,714 & 272 & $0.9(0.81-1.11)$ \\
\hline 4000-4999 & 9770 & 133 & $0.9(0.75-1.14)$ & 1049 & 22 & $1.4(0.83-2.52)$ & 9895 & 155 & $1.0(0.80-1.17)$ \\
\hline$\geq 5000$ & 8559 & 139 & $1.0(0.84-1.28)$ & 907 & 24 & $1.5(0.87-2.71)$ & 8700 & 163 & $1.1(0.88-1.30)$ \\
\hline \multicolumn{10}{|c|}{ Potassium $(\mathrm{mg})^{3}$} \\
\hline$<1400$ & 8840 & 158 & $1.0(0.76-1.30)$ & 7903 & 35 & $1.5(0.53-4.05)$ & 9022 & 193 & $1.0(0.79-1.29)$ \\
\hline $1400-2400$ & 29,603 & 471 & $1.1(0.86-1.32)$ & 26,620 & 39 & $0.7(0.30-1.57)$ & 29,810 & 510 & $1.0(0.81-1.20)$ \\
\hline $2400-3400$ & 22,361 & 324 & $1.1(0.93-1.35)$ & 20,383 & 46 & $1.4(0.70-2.20)$ & 22,602 & 370 & $1.1(0.93-1.32)$ \\
\hline$\geq 3400$ & 13,602 & 187 & 1.0 & 12,335 & 33 & 1.0 & 13,782 & 220 & 1.0 \\
\hline \multicolumn{10}{|l|}{$\operatorname{Iron}(\mathrm{mg})^{3}$} \\
\hline$<7$ & 14,913 & 272 & $1.0(0.85-1.18)$ & 13,250 & 48 & $1.9(1.22-3.10)$ & 15,164 & 320 & $1.1(0.91-1.24)$ \\
\hline $7-10$ & 21,142 & 340 & 1.0 & 18,986 & 31 & 1.0 & 21,303 & 371 & 1.0 \\
\hline $10-15$ & 26,076 & 369 & $1.0(0.81-1.17)$ & 23,763 & 44 & $1.5(0.87-2.62)$ & 26,315 & 413 & $1.0(0.85-1.20)$ \\
\hline$\geq 15$ & 12,274 & 159 & $0.9(0.69-1.12)$ & 11,241 & 30 & $2.1(1.06-4.34)$ & 12,434 & 189 & $1.0(0.77-1.21)$ \\
\hline \multicolumn{10}{|l|}{ Zinc (mg) ${ }^{3}$} \\
\hline$<5$ & 6484 & 143 & $0.92(0.71-1.21)$ & 5620 & 32 & $2.5(1.23-5.13)$ & 6637 & 175 & $1.0(0.80-1.31)$ \\
\hline $5-8$ & 30,145 & 478 & $0.9(0.69-1.05)$ & 27,221 & 57 & $1.3(0.68-2.28)$ & 30,453 & 535 & $0.9(0.73-1.08)$ \\
\hline $8-11$ & 23,234 & 339 & 1.0 & 21,044 & 38 & 1.0 & 23,448 & 377 & 1.0 \\
\hline$\geq 11$ & 14,543 & 180 & $0.8(0.69-1.00)$ & 13,355 & 26 & $0.9(0.56-1.58)$ & 14,678 & 206 & $0.8(0.71-1.01)$ \\
\hline \multicolumn{10}{|c|}{ Vitamin A (R.E) ${ }^{3}$} \\
\hline$<300$ & 19,067 & 368 & $1.1(0.94-1.39)$ & 16,828 & 54 & $1.0(0.59-1.68)$ & 19,333 & 422 & $1.1(0.93-1.35)$ \\
\hline $300-500$ & 24,116 & 360 & $1.1(0.89-1.28)$ & 21,814 & 38 & $0.7(0.43-1.13)$ & 24,309 & 398 & $1.0(0.87-1.21)$ \\
\hline $500-700$ & 14,441 & 198 & $1.1(0.89-1.31)$ & 13,124 & 24 & $1.0(0.60-1.70)$ & 14,567 & 222 & $1.1(0.89-1.28)$ \\
\hline$\geq 700$ & 16,783 & 214 & 1.0 & 15,475 & 37 & 1.0 & 17,006 & 251 & 1.0 \\
\hline \multicolumn{10}{|l|}{ Retinol $(\mu g)^{3}$} \\
\hline$<20$ & 13,540 & 300 & $1.1(0.86-1.31)$ & 11,714 & 62 & $2.2(1.23-3.93)$ & 13,849 & 362 & $1.2(0.95-1.40)$ \\
\hline $20-60$ & 25,553 & 402 & $1.2(0.96-1.39)$ & 22,939 & 43 & $1.4(0.79-2.46)$ & 25,765 & 445 & $1.2(0.99-1.41)$ \\
\hline $60-100$ & 18,528 & 243 & $1.1(0.90-1.32)$ & 17,034 & 29 & $1.3(0.72-2.34)$ & 18,707 & 272 & $1.1(0.93-1.34)$ \\
\hline$\geq 100$ & 16,784 & 195 & 1.0 & 15,553 & 19 & 1.0 & 16,895 & 214 & 1.0 \\
\hline \multicolumn{10}{|c|}{ Carotene $(\mu \mathrm{g})^{3}$} \\
\hline$<1200$ & 13,517 & 248 & $1.0(0.82-1.23)$ & 11,995 & 34 & $0.9(0.53-1.58)$ & 13,685 & 282 & $1.0(0.83-1.21)$ \\
\hline $1200-2300$ & 27,116 & 430 & $1.1(0.91-1.27)$ & 24,502 & 53 & $0.8(0.54-1.33)$ & 27,381 & 483 & $1.1(0.90-1.24)$ \\
\hline $2300-3400$ & 15,849 & 224 & $1.0(0.87-1.26)$ & 14,282 & 29 & $1.1(0.67-1.81)$ & 16,007 & 253 & $1.0(0.88-1.25)$ \\
\hline$\geq 3400$ & 17,924 & 238 & 1.0 & 16,461 & 37 & 1.0 & 18,144 & 275 & 1.0 \\
\hline \multicolumn{10}{|c|}{ Vitamin $\mathrm{E}(\mathrm{mg})^{3}$} \\
\hline$<5$ & 11,027 & 206 & $1.0(0.81-1.33)$ & 9817 & 39 & $1.1(0.52-1.94)$ & 11,209 & 245 & $1.0(0.83-1.31)$ \\
\hline $5-8$ & 22,751 & 400 & $1.3(1.03-1.55)$ & 20,213 & 35 & $0.7(0.37-1.24)$ & 22,950 & 435 & $1.2(0.97-1.43)$ \\
\hline $8-11$ & 19,223 & 271 & $1.1(0.94-1.34)$ & 17,543 & 40 & $1.0(0.65-1.70)$ & 19,429 & 311 & $1.1(0.95-1.32)$ \\
\hline$\geq 11$ & 21,404 & 263 & 1.0 & 19,667 & 39 & 1.0 & 21,628 & 302 & 1.0 \\
\hline
\end{tabular}


Table 1. Cont.

\begin{tabular}{|c|c|c|c|c|c|c|c|c|c|}
\hline & \multirow{2}{*}{$\begin{array}{l}\text { Person- } \\
\text { Years }\end{array}$} & \multicolumn{2}{|c|}{$\begin{array}{c}\text { CKD Stage } 3 A^{1} \\
\quad(n=1140)\end{array}$} & \multirow{2}{*}{$\begin{array}{c}\text { Person- } \\
\text { Years }\end{array}$} & \multicolumn{2}{|c|}{$\begin{array}{l}\text { CKD Stage 3B and over }{ }^{1} \\
\qquad(n=153)\end{array}$} & \multirow{2}{*}{$\begin{array}{l}\text { Person- } \\
\text { Years }\end{array}$} & \multicolumn{2}{|c|}{$\begin{array}{c}\text { CKD } \\
(n=1293)\end{array}$} \\
\hline & & $\begin{array}{c}\text { Cases } \\
n\end{array}$ & $\begin{array}{c}\text { HR } \\
(95 \% \mathrm{CI})^{2}\end{array}$ & & $\begin{array}{c}\text { Cases } \\
n\end{array}$ & $\begin{array}{c}\text { HR } \\
(95 \% \text { CI })^{2}\end{array}$ & & $\begin{array}{c}\text { Cases } \\
n\end{array}$ & $\begin{array}{c}\text { HR } \\
(95 \% \text { CI })^{2}\end{array}$ \\
\hline \multicolumn{10}{|c|}{ Vitamin B1 $(\mathrm{mg})^{3}$} \\
\hline$<0.9$ & 17,773 & 339 & $1.0(0.85-1.18)$ & 15,727 & 51 & $1.3(0.79-1.96)$ & 18,038 & 390 & $1.0(0.87-1.19)$ \\
\hline $0.9-1.2$ & 18,463 & 306 & 1.0 & 16,625 & 35 & 1.0 & 18,645 & 341 & 1.0 \\
\hline $1.2-1.5$ & 19,236 & 252 & $0.9(0.77-1.14)$ & 17,620 & 32 & $1.3(0.71-2.34)$ & 19,404 & 284 & $1.0(0.79-1.15)$ \\
\hline$\geq 1.5$ & 18,934 & 243 & $0.8(0.66-1.04)$ & 17,269 & 35 & $1.2(0.58-2.42)$ & 19,128 & 278 & $0.9(0.69-1.06)$ \\
\hline \multicolumn{10}{|c|}{ Vitamin B2 $(\mathrm{mg})^{3}$} \\
\hline$<0.7$ & 17,674 & 361 & $1.0(0.82-1.28)$ & 15,478 & 64 & $2.3(1.18-4.45)$ & 18,004 & 425 & $1.1(0.89-1.35)$ \\
\hline $0.7-0.9$ & 18,651 & 294 & $1.0(0.85-1.26)$ & 16,859 & 31 & $1.4(0.78-2.60)$ & 18,811 & 325 & $1.1(0.88-1.28)$ \\
\hline $0.9-1.2$ & 19,248 & 263 & 1.0 & 17,502 & 25 & 1.0 & 19,381 & 288 & 1.0 \\
\hline$\geq 1.2$ & 18,833 & 222 & $0.9(0.78-1.13)$ & 17,401 & 33 & $1.2(0.69-2.02)$ & 19,021 & 255 & $1.0(0.82-1.16)$ \\
\hline \multicolumn{10}{|c|}{ Niacin $(m g)^{3}$} \\
\hline$<10$ & 17,808 & 345 & $0.7(0.58-1.00)$ & 10,508 & 44 & $1.1(0.58-2.28)$ & 18,116 & 404 & $0.8(0.61-0.98)$ \\
\hline $10-14$ & 18,611 & 300 & $0.9(0.69-1.06)$ & 19,311 & 42 & $0.8(0.44-1.50)$ & 18,786 & 333 & $0.9(0.70-1.06)$ \\
\hline $14-19$ & 18,891 & 271 & 1.0 & 20,904 & 40 & 1.0 & 19,059 & 303 & 1.0 \\
\hline$\geq 19$ & 19,095 & 224 & $0.9(0.76-1.09)$ & 16,517 & 27 & $1.0(0.57-1.60)$ & 19,255 & 253 & $0.9(0.77-1.09)$ \\
\hline \multicolumn{10}{|c|}{ Folate $(\mu g)^{3}$} \\
\hline$<100$ & 3555 & 57 & $1.1(0.75-1.54)$ & 3248 & 22 & $3.0(1.04-8.39)$ & 3663 & 79 & $1.2(0.89-1.68)$ \\
\hline $100-200$ & 25,961 & 433 & $1.2(0.97-1.60)$ & 23,253 & 47 & $1.1(0.45-2.63)$ & 26,224 & 480 & $1.2(0.93-1.49)$ \\
\hline $200-300$ & 26,083 & 396 & $1.2(0.96-1.51)$ & 23,536 & 36 & $0.7(0.35-1.45)$ & 26,255 & 432 & $1.1(0.91-1.40)$ \\
\hline $300-400$ & 11,099 & 153 & $1.2(0.94-1.56)$ & 10,177 & 28 & $1.4(0.75-2.50)$ & 11,244 & 181 & $1.2(0.98-1.55)$ \\
\hline$\geq 400$ & 7708 & 101 & 1.0 & 7027 & 20 & 1.0 & 7830 & 121 & 1.0 \\
\hline \multicolumn{10}{|c|}{ Vitamin B6 $(\mathrm{mg})^{3}$} \\
\hline$<1.0$ & 5946 & 120 & $1.0(0.80-1.25)$ & 5292 & 30 & $2.7(1.52-4.93)$ & 6078 & 150 & $1.1(0.91-1.37)$ \\
\hline $1.0-1.3$ & 12,436 & 215 & $1.0(0.86-1.25)$ & 11,058 & 22 & $1.2(0.65-2.25)$ & 12,567 & 237 & $1.0(0.87-1.24)$ \\
\hline $1.3-1.6$ & 15,251 & 244 & 1.0 & 13,729 & 19 & 1.0 & 15,349 & 263 & 1.0 \\
\hline$\geq 1.6$ & 40,773 & 561 & $1.0(0.82-1.20)$ & 37,161 & 82 & $2.3(1.24-4.14)$ & 41,222 & 643 & $1.1(0.89-1.28)$ \\
\hline \multicolumn{10}{|c|}{ Vitamin C $(\mathrm{mg})^{3}$} \\
\hline$<60$ & 13,034 & 215 & $0.9(0.77-1.14)$ & 11,815 & 30 & $1.4(0.77-2.61)$ & 13,176 & 245 & $1.0(0.81-1.17)$ \\
\hline $60-75$ & 8369 & 145 & $1.0(0.84-1.29)$ & 7459 & 17 & $1.7(0.83-3.33)$ & 8472 & 162 & $1.1(0.87-1.31)$ \\
\hline $75-100$ & 13,717 & 208 & 1.0 & 12,418 & 16 & 1.0 & 13,802 & 224 & 1.0 \\
\hline$\geq 100$ & 39,286 & 572 & $0.9(0.79-1.11)$ & 35,547 & 90 & $1.9(1.07-3.22)$ & 39,766 & 662 & $1.0(0.85-1.17)$ \\
\hline
\end{tabular}

CKD, chronic kidney disease; KoGES, Korea Genome and Epidemiologic Study; eGFR, estimated glomerular filtration rate; CKD-EPI, Chronic Kidney Disease Epidemiology Collaboration. ${ }^{1}$ CKD was defined as an eGFR $<60 \mathrm{~mL} / \mathrm{min} / 1.73 \mathrm{~m}^{2}$ by the CKD-EPI criteria. Stage $3 \mathrm{~A}$ and stage $3 \mathrm{~B}$ and over were defined as $45 \leq$ eGFR $<60$ and eGFR $<45$, respectively. ${ }^{2}$ Cox proportional hazard model adjusted for age, sex, baseline eGFR, body mass index (BMI), regular physical activity, cigarette smoking, alcohol consumption, total cholesterol level in blood, hypertension, diabetes, the urine albumin to creatinine ratio, uric acid in urine, energy intake (mg/day), and protein intake (mg/day); for the analysis of 'potassium intake', additionally adjusted for folate intake/day. ${ }^{3}$ For calcium, RDA (recommended dietary allowance for the general population) $=1300 \mathrm{mg}$; DRI (dietary reference intakes by KDOQI Clinical practice guideline for CKD patients or dialyzed CKD patients) $=800 \mathrm{mg}$ (women CKD), $1000 \mathrm{mg}$ (men CKD); for phosphorus, $\mathrm{RDA}=700 \mathrm{mg}$; DRI $=400-700 \mathrm{mg}$ (CKD); for sodium, AI (adequate intake for the general population) $=2000 \mathrm{mg}$; DRI = limit or reduce sodium intake (CKD); for potassium, $\mathrm{AI}=3400 \mathrm{mg}$; DRI = adjust dietary potassium intake to maintain serum potassium within the normal range (CKD) and reduce dietary potassium intake (e.g., $2000 \mathrm{mg}$ ) (CKD 3-5 with hyperkalemia); for iron, RDA $=8 \mathrm{mg}$ (women with age $\geq 51$ or adult men), $=18 \mathrm{mg}$ (women with age 19-50); DRI = $10 \mathrm{mg}$ (CKD). CKD patients need regular blood tests for iron overload; for zinc, RDA = $8 \mathrm{mg}$ (women); DRI = $8 \mathrm{mg}$ (women CKD); $10 \mathrm{mg}$ (men CKD) $15 \mathrm{mg}$ (dialysis); for vitamin A, RDA = $900 \mathrm{mg}$; DRI = 700-900 mg (CKD); for vitamin A precursor (retinol and carotene, not defined RDA or DRI); for vitamin E, RDA = $11 \mathrm{mg}$ (women) $15 \mathrm{mg}$ (men), DRI = more than RDA (CKD); for vitamin $\mathrm{B} 1, \mathrm{RDA}=1.1 \mathrm{mg}, \mathrm{DRI}=1.5 \mathrm{mg}(\mathrm{CKD})$; for vitamin B2, RDA $=1.1 \mathrm{mg}$ (women) $1.3 \mathrm{mg}$ (men), DRI: $1.8 \mathrm{mg}$ on a low-protein diet (CKD), 1.1-1.3 mg (dialysis), especially with poor appetite; for niacin, RDA = $14 \mathrm{mg}$ (women) $16 \mathrm{mg}$ (men); DRI = near to RDA (dialysis); for folate, $\mathrm{RDA}=400 \mu \mathrm{g}$, DRI = more than RDA (CKD); for vitamin B6, RDA = $1.3 \mathrm{mg}$, DRI = $2 \mathrm{mg}$ (dialysis); for vitamin C, RDA $=75 \mathrm{mg}$ (women) $90 \mathrm{mg}$ (men); DRI = 60-100 mg (dialysis).

When the vitamin intake was divided into categories, the lowest and highest intake levels were associated with increased risk of CKD stage 3B and over compared with the reference levels, which included RDA, AI, or National Kidney Foundation guideline values for retinol $(<20 \mu \mathrm{g} /$ day, $\mathrm{HR}=2.2,95 \%$ CI: 1.23-3.93), vitamin B2 $(<0.7 \mathrm{mg} /$ day, $\mathrm{HR}=2.3,95 \%$ CI: 1.18-4.45), folate $(<100 \mu \mathrm{g} /$ day, HR $=3.0,95 \%$ CI: 1.04-8.39), vitamin B6 $(<1.0 \mathrm{mg} /$ day, $\mathrm{HR}=2.7,95 \%$ CI: $1.52-4.93 ; \geq 1.6 \mathrm{mg} /$ day, $\mathrm{HR}=2.3,95 \% \mathrm{CI}: 1.12-4.66)$, and vitamin C ( $\geq 100 \mathrm{mg} /$ day, HR $=2.3,95 \%$ CI: 1.24-4.14) (Table 1$)$.

In addition, these results were consistent in the sensitivity analyses in the restricted cohort population ( $n=8901)$ excluding newly defined CKD cases within 2 years from study entry (Supplementary Table S3). In the risk of diabetes and hypertensive CKD, although the strength of the association was slightly reduced due to the decrease in the number of cases, the direction of the association remained the same (Supplementary Table S4). None of the micronutrients showed a significant association with the risk of developing CKD 
stage 3A (Entire cohort, Table 1; Restricted cohort, Supplementary Table S3; For diabetes and hypertensive CKD 3A). The association between overall CKD risk and dietary factors was observed as a null effect. This was a result of reflecting CKD stage 3A because new cases being CKD stage 3A accounted for $88.2 \%$ out of all new CKD cases and the effect size of CKD stage 3A was similar to that of all cases (Table 1, Supplementary Table S3).

\subsection{Discriminatory Accuracy of Models on Predicting the Risk of CKD 3B and over}

Figure 2 shows the discriminant ability of each model to distinguish between new cases of CKD and non-cases over 12 year follow-up periods in the cohort population. With the ability to discriminate new CKD cases for the clinico-nutritional model, a multivariate model controlled by various micronutrients and other risk factors, the Harrell's C-index at long-term follow-up was as follows: Model 1 (dietary nutritional) was constructed with 10 variables and the Harrell's C-index was 0.7341. Model 2 (clinico-epidemiological) was constructed with 8 variables and the Harrell's C-index was 0.9225 . Model 3 (cliniconutritional) was built using all variables in model 1 plus model 2 and the Harrell's C-index was 0.9371 . The clinico-nutritional model, a full-multivariable model, showed the highest differential accuracy among the three models predicting the risk of developing CKD stage 3B or higher. The C-index of the model was significantly different from that of the clinicoepidemiological model $(p<0.01)$.

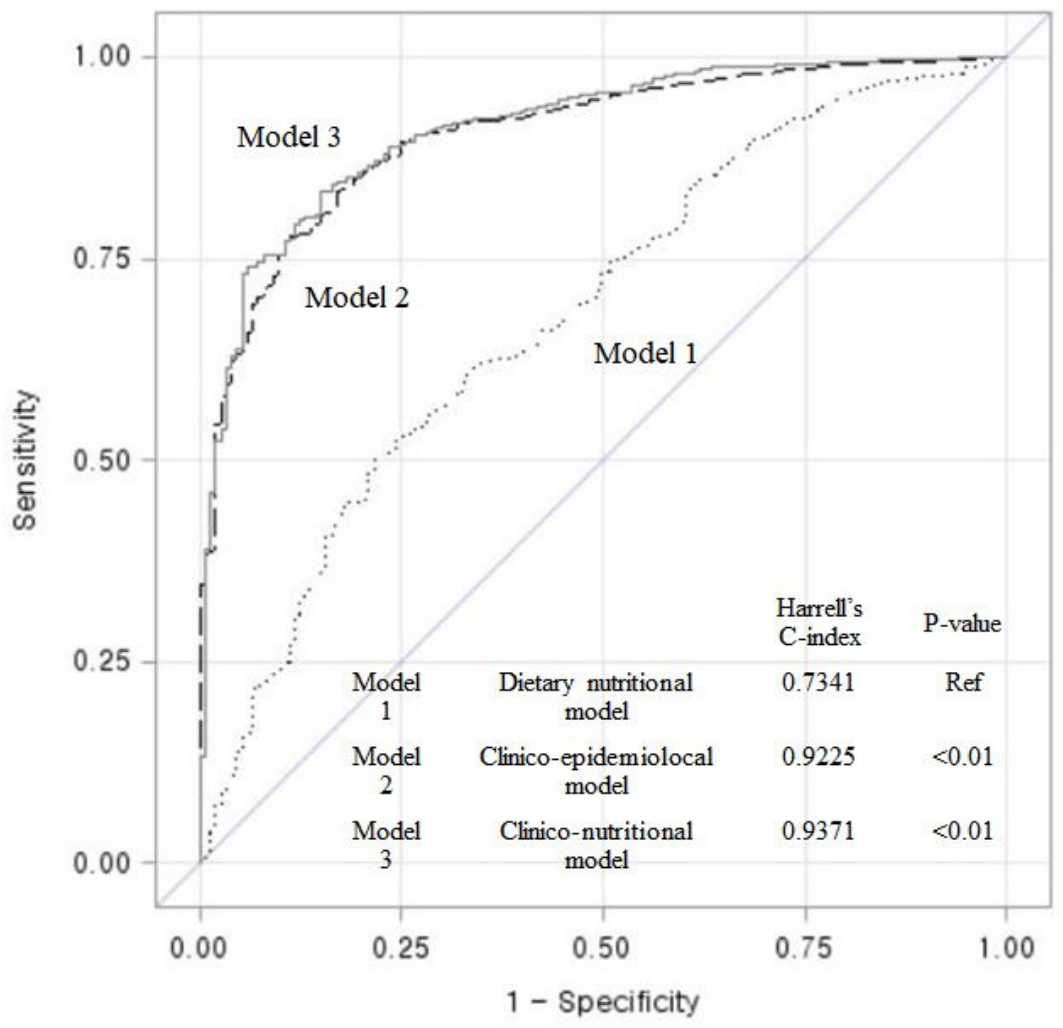

Figure 2. Receiver operating characteristic (ROC) curves and Harrell's C-index showing the discriminant accuracy of each model for the ability to distinguish new cases of CKD stage 3B and over in the entire cohort population over 12 year follow-up periods. CKD, chronic kidney disease. Model 1 (dietary nutritional model): Function $(Y)=\beta_{1}[$ Calcium $]+\beta_{2}[$ Phosphorus $]+\beta_{3}[$ Sodium $]+\beta_{4}[$ Iron $]+$ $\beta_{5}[$ Retinol $]+\beta_{6}\left[\right.$ Vitamin B2] $+\beta_{7}[$ Folate $]+\beta_{8}\left[\right.$ Vitamin B6] $+\beta_{9}[$ Vitamin C $]+\beta_{10}[$ Total Calories $]+$ $\beta_{11}$ [Protein], Function $(Y)=\log \left(\frac{\text { Hazard }_{\text {Exposed }}}{\text { Hazard }_{\text {Non-Exposed }}}\right)$; Model 2 (clinico-epidemiological model): Func$\operatorname{tion}(Y)=\beta_{1}[$ Age $]+\beta_{2}[$ Sex $]+\beta_{3}[$ Baseline eGFR $]+\beta_{4}[$ Physical activity $]+\beta_{5}[$ Cigarette smoking] $+\beta_{6}[$ Hypertension $]+\beta_{7}[$ Diabetes $]+\beta_{8}$ [Body mass index]; Model 3 (clinico-nutritional model, a composite model of Model 1 and Model 2): Function $(Y)=\beta_{11}^{1}\left[\right.$ Model 1] $+\beta_{8}^{1}$ [Model 2]). 
The discriminant ability of the clinico-nutritional model including multiple micronutrients and risk factors to distinguish between new cases of diabetic and hypertensive CKD stage 3B and over and non-cases was also high (C-index 0.8989 and 0.9296, respectively) (Supplementary Figure S1). We did not construct a clinico-nutritional model and a dietary nutritional model due to non-significant results in the association between any micronutrients and the risk of CKD stage 3A.

\subsection{Micronutrient Intake for the Risk of CKD Stage $3 B$ and over in a Full Multivariable Clinico-Nutritional Model}

Table 2 shows the adjusted HRs for the risk of CKD stage 3B and over associated with micronutrients in the full multivariable model (clinico-nutritional model) controlled by multiple micronutrients and other risk factors. Low phosphorus and vitamin B2 and high vitamins $B 6$ and $C$ intakes were positively associated with the risk of developing $C K D$ stage $3 \mathrm{~B}$ and over, respectively (phosphorus $<400 \mathrm{mg}$ /day, $\mathrm{HR}=6.78,95 \% \mathrm{CI}$ : 2.18-21.11; vitamin $\mathrm{B} 2<0.7 \mathrm{mg} /$ day, $\mathrm{HR}=2.90,95 \% \mathrm{CI}$ : 1.01-8.33; vitamin B6 $\geq 1.6 \mathrm{mg} /$ day, $\mathrm{HR}=2.71$, 95\% CI: $1.26-5.81$; vitamin C $\geq 100 \mathrm{mg} /$ day, $\mathrm{HR}=1.83,95 \% \mathrm{CI}: 1.00-3.33)$. Outside of the nutritional factors, age (HR: 1.17, 95\% CI: 1.14-1.21), baseline eGFR (HR: 0.94, 95\% CI: 0.93-0.95; $p<0.01$ ), female (HR: 2.14, 95\% CI: 1.21-3.79), smoking (HR: 1.96, 95\% CI: 1.11-3.45), HTN (HR: 1.71, 95\% CI: 1.23-2.38), DM (HR: 5.01, 95\% CI: 3.51-7.15; $p<0.01$ ) were shown to be statistically significant. Sensitivity analysis was performed in the restricted population excluding CKD cases that newly occurred within 2 years. An additional association with low folate levels ( $<100 \mu \mathrm{g} /$ day) in higher risk of CKD stage 3B and over was observed (HR: 6.72, 95\% CI: 1.40-32.16). Moreover, a much stronger association was observed with phosphorus intake. $(<400 \mathrm{mg} /$ day, HR: $8.51,95 \% \mathrm{CI}$ : 2.33-31.08; for 400-700 mg/day, HR: 2.42, 95\% CI: 1.02-5.74).

Table 2. Micronutrients and $\mathrm{CKD}^{1}$ risk stage $3 \mathrm{~B}$ and over in the full multivariable model (clinico-nutritional model) ${ }^{2}$ controlling multiple nutrients and additional risk factors.

\begin{tabular}{|c|c|c|c|c|}
\hline & \multicolumn{2}{|c|}{ Entire Cohort } & \multicolumn{2}{|c|}{ Restricted Cohort $^{3}$} \\
\hline & HR $(95 \% \text { CI })^{2}$ & $p$-Value & HR $(95 \% \text { CI })^{2}$ & $p$-Value \\
\hline Age & $1.17(1.14-1.21)$ & $<0.01$ & $1.17(1.14-1.22)$ & $<0.01$ \\
\hline Sex (Female) & $2.14(1.21-3.79)$ & $<0.01$ & $1.96(1.06-3.64)$ & 0.03 \\
\hline Baseline eGFR & $0.94(0.93-0.95)$ & $<0.01$ & $0.95(0.94-0.94)$ & $<0.01$ \\
\hline Smoker & $1.96(1.11-3.45)$ & 0.02 & $1.54(0.83-2.84)$ & 0.17 \\
\hline Physical activity & $1.20(0.85-1.68)$ & 0.29 & $1.08(0.12-2.05)$ & 0.67 \\
\hline $\mathrm{BMI}(\geq 25)$ & $1.27(0.90-1.79)$ & 0.17 & $1.19(0.82-1.72)$ & 0.36 \\
\hline Hypertension & $1.71(1.23-2.38)$ & $<0.01$ & $1.79(1.24-2.56)$ & $<0.01$ \\
\hline Diabetes & $5.01(3.51-7.15)$ & $<0.01$ & $5.46(3.71-8.03)$ & $<0.01$ \\
\hline Diet energy intake & $0.94(0.56-1.59)$ & 0.82 & $0.94(0.54-1.65)$ & 0.83 \\
\hline Diet protein intake & $1.41(0.71-2.78)$ & 0.32 & $1.43(0.69-2.95)$ & 0.33 \\
\hline \multicolumn{5}{|l|}{ Calcium $(\mathrm{mg})^{4}$} \\
\hline$<200$ & $0.68(0.21-2.20)$ & 0.51 & $0.77(0.21-2.85)$ & 0.69 \\
\hline $200-400$ & $0.84(0.34-2.05)$ & 0.69 & $0.98(0.37-2.60)$ & 0.97 \\
\hline $400-600$ & $1.06(0.54-2.08)$ & 0.86 & $1.21(0.58-2.55)$ & 0.60 \\
\hline$\geq 600$ & 1.0 & - & 1.0 & - \\
\hline \multicolumn{5}{|l|}{ Phosphorus (mg) 4} \\
\hline$<400$ & $6.78(2.18-21.11)$ & $<0.01$ & $8.51(2.33-31.08)$ & $<0.01$ \\
\hline $400-700$ & $1.89(0.95-4.11)$ & 0.09 & $2.42(1.02-5.74)$ & 0.04 \\
\hline $700-1200$ & 1.0 & - & 1.0 & - \\
\hline$\geq 1200$ & $0.62(0.29-1.36)$ & 0.23 & $0.60(0.26-1.37)$ & 0.22 \\
\hline \multicolumn{5}{|l|}{ Sodium (mg) ${ }^{4}$} \\
\hline$<2000$ & $0.95(0.54-1.69)$ & 0.86 & $0.95(0.50-1.82)$ & 0.88 \\
\hline 2000-2999 & 1.0 & - & 1.0 & - \\
\hline 3000-3999 & 1.18 (0.70-1.99) & 0.54 & $1.14(0.64-2.04)$ & 0.65 \\
\hline $4000-4999$ & $1.25(0.67-2.32)$ & 0.47 & $1.45(0.75-2.83)$ & 0.26 \\
\hline$\geq 5000$ & $1.38(0.69-2.76)$ & 0.36 & $1.76(0.84-3.68)$ & 0.13 \\
\hline
\end{tabular}


Table 2. Cont.

\begin{tabular}{|c|c|c|c|c|}
\hline & \multicolumn{2}{|c|}{ Entire Cohort } & \multicolumn{2}{|c|}{ Restricted Cohort $^{3}$} \\
\hline & HR $\left(95 \%\right.$ CI) ${ }^{2}$ & $p$-Value & HR $(95 \% \text { CI })^{2}$ & $p$-Value \\
\hline \multicolumn{5}{|c|}{ Iron $(\mathrm{mg})^{4}$} \\
\hline$<7$ & $1.00(0.46-2.17)$ & 0.99 & $0.93(0.41-2.15)$ & 0.87 \\
\hline $7-10$ & 1.0 & - & 1.0 & - \\
\hline 10-15 & $1.11(0.58-2.13)$ & 0.74 & $1.22(0.60-2.51)$ & 0.57 \\
\hline$\geq 15$ & $2.05(0.72-5.82)$ & 0.17 & $2.06(0.66-6.46)$ & 0.21 \\
\hline \multicolumn{5}{|c|}{ Retinol $(\mu \mathrm{g})^{4}$} \\
\hline$<20$ & $1.78(0.81-3.92)$ & 0.15 & $1.78(0.76-4.16)$ & 0.18 \\
\hline $20-60$ & $1.28(0.63-2.62)$ & 0.49 & $1.24(0.57-2.68)$ & 0.59 \\
\hline $60-100$ & $1.25(0.67-2.34)$ & 0.47 & $1.33(0.68-2.61)$ & 0.40 \\
\hline$\geq 100$ & 1.0 & - & 1.0 & - \\
\hline \multicolumn{5}{|c|}{ Vitamin B2 (mg) ${ }^{4}$} \\
\hline$<0.7$ & $2.90(1.01-8.33)$ & 0.04 & $2.69(0.95-8.10)$ & 0.08 \\
\hline $0.7-0.9$ & $1.81(0.87-3.74)$ & 0.11 & $2.13(0.98-4.64)$ & 0.05 \\
\hline $0.9-1.2$ & 1.0 & - & 1.0 & - \\
\hline$\geq 1.2$ & $0.92(0.43-1.95)$ & 0.81 & $1.11(0.49-2.55)$ & 0.06 \\
\hline \multicolumn{5}{|c|}{ Folate $(\mu \mathrm{g})^{4}$} \\
\hline$<100$ & $2.57(0.66-10.08)$ & 0.17 & $6.72(1.40-32.16)$ & 0.02 \\
\hline $100-200$ & $1.39(0.48-4.04)$ & 0.54 & $1.35(0.44-4.19)$ & 0.60 \\
\hline $200-300$ & $0.74(0.33-1.70)$ & 0.48 & $0.60(0.25-1.44)$ & 0.25 \\
\hline $300-400$ & $1.38(0.71-2.70)$ & 0.34 & $1.09(0.53-2.23)$ & 0.75 \\
\hline$\geq 400$ & 1.0 & - & 1.0 & - \\
\hline \multicolumn{5}{|c|}{ Vitamin B6 (mg) 4} \\
\hline$<1.0$ & $0.90(0.32-2.56)$ & 0.84 & $0.43(0.12-1.55)$ & 0.19 \\
\hline $1.0-1.3$ & $0.87(0.42-1.81)$ & 0.71 & $0.97(0.44-2.14)$ & 0.93 \\
\hline $1.3-1.6$ & 1.0 & - & 1.0 & - \\
\hline$\geq 1.6$ & $2.71(1.26-5.81)$ & 0.01 & $3.08(1.34-7.09)$ & $<0.01$ \\
\hline \multicolumn{5}{|c|}{ Vitamin C (mg) ${ }^{4}$} \\
\hline$<60$ & $0.64(0.31-1.32)$ & 0.23 & $0.45(0.19-1.04)$ & 0.06 \\
\hline $60-75$ & $1.39(0.69-2.80)$ & 0.35 & $1.45(0.68-3.06)$ & 0.33 \\
\hline $75-100$ & 1.0 & - & 1.0 & - \\
\hline$\geq 100$ & $1.83(1.00-3.33)$ & 0.05 & $1.79(0.98-3.41)$ & 0.08 \\
\hline
\end{tabular}

CKD, chronic kidney disease; KoGES, Korea Genome and Epidemiologic Study; eGFR, estimated glomerular filtration rate; CKD-EPI, Chronic Kidney Disease Epidemiology Collaboration. ${ }^{1}$ CKD was defined as an eGFR $<60 \mathrm{~mL} / \mathrm{min} / 1.73 \mathrm{~m}^{2}$ by the CKD-EPI criteria. Stage 3B and over was defined as an eGFR $<45$, respectively. ${ }^{2}$ The clinico-nutritional model was a multivariable Cox proportional hazard model, constructed as Function $(\mathrm{Y})=\sum_{i}^{n} \beta \mathrm{i}[$ all variables $]$ which were listed in the table; Function $(\mathrm{Y})=\log \left(\frac{\text { Hazard }}{\text { Hazard } \text { Exposed }}\right)$ - xxposed $) .{ }^{3}$ Restricted cohort analysis excluding new CKD cases within 2 years from cohort entry. ${ }^{4}$ For calcium, RDA (recommended dietary allowance for the general population) $=1300 \mathrm{mg}$; DRI (dietary reference intakes by KDOQI Clinical practice guideline for CKD patients or dialyzed CKD patients) $=800 \mathrm{mg}$ (women CKD), $1000 \mathrm{mg}$ (men CKD); for phosphorus, RDA = 700 mg; DRI = 400-700 mg (CKD); for sodium, AI (adequate intake for the general population) $=2000 \mathrm{mg}$; DRI = limit or reduce sodium intake (CKD); for iron, RDA = $8 \mathrm{mg}$ (women with age $\geq 51$ or adult men), $=18 \mathrm{mg}$ (women with age 19-50); DRI $=10 \mathrm{mg}$ (CKD). CKD patients need regular blood tests for iron overload; for vitamin A precursor (retinol and carotene, not defined RDA or DRI); for vitamin B2, RDA =1.1 mg (women) $1.3 \mathrm{mg}$ (men), DRI: $1.8 \mathrm{mg}$ on a low-protein diet (CKD), 1.1-1.3 mg (dialysis), especially with poor appetite; for folate, $\mathrm{RDA}=400 \mu \mathrm{g}$, DRI = More than RDA (CKD); for vitamin B6, RDA = $1.3 \mathrm{mg}, \mathrm{DRI}=2 \mathrm{mg}$ (dialysis); for vitamin C, RDA = $75 \mathrm{mg}$ (women) $90 \mathrm{mg}$ (men); DRI = 60-100 mg (dialysis).

In addition, the association with the selected micronutrients and clinico-epidemiological factors on the risk of diabetic or hypertensive CKD stage 3B and over were persistent (Supplementary Table S5). None of the micronutrients showed a significant association with the risk of developing CKD stage 3A.

\section{Discussion}

In this study, we found that, among the general population, low dietary intakes of phosphorus, vitamin B2 (riboflavin), and folate and high dietary intake of vitamins B6 and $C$ were associated with an increased risk of CKD stage $3 B$ and over, compared with the intake at recommended levels in the multivariable Cox model adjusted for multiple 
micronutrients and other clinico-epidemiological risk factors. None of the categories of the minerals/vitamins showed a significant association with the risk of developing CKD stage 3A.

Dietary restriction of phosphorus is recommended for CKD patients as a means of managing hyperphosphatemia because hyperphosphatemia is associated with kidney failure in CKD patients $[17,18]$. A prospective CKD patient cohort study suggested that hemodialysis patients (HD) must ingest no more than $800 \mathrm{mg} /$ day of phosphorus [19]. It is correct to recommend low phosphorus intake for CKD patients. Our study group was not diagnosed with CKD at baseline and also had an eGFR of 60 or higher. When two of them were defined as a new CKD case two years later, phosphorus intakes $<400 \mathrm{mg}$ in the entire population (even phosphorus intakes $<700 \mathrm{mg}$ in the restricted population after excluding new $\mathrm{CKD}$ cases within 2 years) were associated with the risk of CKD stage $3 \mathrm{~B}$ and over. Of CKD stage 3A patients, those with low phosphorus intake below $400 \mathrm{mg}$ and below $700 \mathrm{mg}$ accounts for $1.9 \%$ and $24.2 \%$, respectively, but of patients being CKD stage $3 \mathrm{~B}$ and over, those accounts for $9.2 \%$ and $34.6 \%$, respectively. In the general population, low phosphorus intake means very low protein intake, i.e., malnutrition, which increases morbidity and mortality [20]. In our previous study, we also reported that low phosphorus intake (<664 mg; 1st quartile) increased the prevalence likelihood of CKD relative to 2nd quartile (664-844 mg) [10]. Low phosphorus intake may reflect that the main food of Koreans is rice and vegetables seasoned with salt and an eating habit of consuming less animal meat and dairy products.

Our previous study is the result of a study based on cross-sectional studies, so the reverse causation acts as a limitation. At the time the study was conducted, follow-up data were not disclosed to researchers. Therefore, despite the limitation of reverse causation in the cross-sectional study, the results were analyzed in the cross-sectional study setting [10]. In contrast, the current study presents causal association in the view of time relevance from exposure to outcome ascertainment because of cohort design showing the risk of CKD new cases. A trend study using repeated cross-sectional studies in Iran, which differs from the actual cohort study design, was conducted. According to that study, the lowest quintile level for phosphorus intake was $820.4 \mathrm{mg}$ (average), and the probability of occurrence of CKD at the highest quintile level compared to the lowest quintile was found to have a null association [7]. We could not directly compare our results with other studies because there is no population that can observe very low phosphate intakes like ours. It is correct to recommend low phosphate intake for CKD patients. However, whether very low phosphate intake is indeed associated with the risk of developing CKD in the general population must be validated, thus requiring large-scale cohort studies involving people with low phosphate intake.

Our results showed that the highest iron intake levels ( $<7 \mathrm{mg} /$ day; $\geq 13.3 \mathrm{mg} /$ day) were associated with an increased risk of CKD stage $3 \mathrm{~B}$ and over compared with the intake at recommended levels (7-10 $\mathrm{mg} /$ day). CKD patients are recommended to consume adequate levels of iron to prevent anemia [21]. For the general population, there were few studies on the association between dietary iron intake and risk of CKD in prospective cohort study. Only a prior cross-sectional study in China showing that iron status was positive correlated with the odds of CKD supports our study result for iron [22]. Biologically, accumulation of iron in the urine or kidney is associated with the onset and progression of the kidney, and excessive iron May also increase the risk of inflammation and damage to free organs [23,24].

Vitamin B2 (riboflavin) status is not routinely measured in a healthy population. The body can store small amounts of riboflavin; therefore, urinary excretion reflects dietary riboflavin intake only after the tissues have been saturated [25]. Although there is no research proving that dietary riboflavin intake is associated with kidney function for the general population, two cross-sectional studies supported our results for riboflavin. The first study reported that low riboflavin and thiamine levels were associated with high fasting total homocysteine (tHcy) plasma levels in ESRD patients, and the second study 
reported a link between high tHcy levels and worse kidney function [26-28]. Vitamin B6 deficiency, like vitamin B2 deficiency, can lead to high levels of tHcy, which can worsen kidney function $[28,29]$. In a multivariable analysis corrected for several micronutrients, the risk of CKD for low B6 intake was observed as a null association, but high B6 intake was still associated with CKD risk. Prior a randomized controlled trial showing that excess vitamin B6 in patients with diabetic nephropathy resulted in a greater decrease in GFR supported our finding for high B6 intake [30]. Additionally, there was no cohort study of the general population directly supporting our findings for an increased risk of CKD at the lowest folic acid intake $(<100 \mu \mathrm{g} /$ day). A clinical trial reported that the simultaneous use of nalapril and folic acid delayed the eGFR deterioration compared to nalapril alone among CKD patients [31]. That result indirectly suggests that low folate levels are associated with high eGFR levels. Given the lack of direct evidence for vitamin B2, vitamin B6 and folic acid, further studies are needed on the association of these intake levels on the risk of developing CKD.

Prior results for vitamin $C$ intake in relation to kidney disease were inconsistent across studies [7,32]. Our result showed that the highest vitamin $C$ intake ( $\geq 100 \mathrm{mg} /$ day) was associated with an increased risk of $\mathrm{CKD}$ stage $3 \mathrm{~B}$ or higher compared to the recommended level (69-103 mg/day). The other cohort study that reported an increased risk of kidney stones compared to the recommended dose ( $<90 \mathrm{mg}$ /day) with higher vitamin C intakes indirectly supported our results [32]. In contrast to our finding, a study from Iran reported the negative association between high intakes of vitamin C and CKD [7]. Although the epidemiological studies are controversial due to inconsistency, the association between high vitamin $C$ intake and eGFR decrease is biologically plausible. Because metabolic by-products of ascorbic acid (i.e., oxalic acid deposits) can accumulate and reduce kidney function, it is recommended that CKD patients do not consume more than $100 \mathrm{mg}$ of vitamin C per day [33].

In our general population, sodium intake is very high. This was the same in the results of a survey that could represent the sodium intake of the entire Korean population (Supplementary Table S1). High sodium intake in CKD patients can accelerate the progression of kidney disease or end-stage renal disease (ESRD) [34,35]. In CKD patients, it is clearly necessary to control sodium intake, but the association with CKD risk has not been clearly demonstrated in the general population of high-dose sodium intake, such as ours. The association between sodium and the risk of developing CKD in other general populations with low sodium intake should be established.

In the case of a single-nutrient analysis, low dietary intakes of calcium, phosphorus, zinc, and retinol were also observed to be associated with an increased risk of CKD. However, in the multivariable model, all of which were corrected for several nutrients, all of these nutrients were not statistically significant. This may be due to the fact that nutrients are consumed together from food, absorbing together or interacting biologically with other nutrients. In the case of zinc, when iron is included in the model, CKD risk and null association were observed, and it could be understood that they are related to each other. Biologically Iron and zinc correlated each other in the case of absorption and a zinc deficiency can coexist with an iron deficiency together [36].

When we analyzed the association with the risk of CKD development based on the average value of dietary factors, no association was observed. However, when we analyzed by classifying them into very low or very high categories and category near the RDA value, dietary intake was observed to be related to CKD risk. This suggests that there is a certain threshold in dietary intake in relation to CKD development and it can be associated with disease only if it is below or above the threshold.

Compared to the RDA of dietary intake, this cohort population tends to consume less calcium, potassium, vitamins A, E, and folic acid and higher sodium and vitamin C. This is the same for the general population (Supplementary Table S1). In addition, cohort members have a high proportion of people who consume less phosphorus and vitamin B2 ( $34.6 \%$ and $41.8 \%$ of all cases above stage $3 \mathrm{~B}$, respectively). Since the main dietary intake 
of Koreans is rice and vegetables seasoned with salt, many nutrients related to fat-soluble vitamins and protein intake tend to be low intake. The nutritional imbalance itself appears to be associated with the eGFR deterioration to CKD stage 3B and over.

Compared with the RDA value of dietary intake, calcium, potassium, vitamin A, E, and folate are ingested less, sodium and vitamin $C$ are ingested a lot, and phosphorus and vitamin B2 are also tended to be low. This is the same for the general population (Supplementary Table S1). As the main dietary intake of Koreans is mainly vegetables including rice and salt, many nutrients related to the intake of fat-soluble vitamins and protein tends to be low intake. The nutritional imbalance itself appears to be associated with the eGFR deterioration to CKD stage 3B and over.

This study had several limitations. First, the definition of the incidence of CKD in this study may lead to overestimation of CKD patients. The definition of new CKD cases in this study was based on a case with decline of eGFR $<60$ after at least two years from the baseline, whereas the diagnostic definition of CKD stage 3A or higher is to find eGFR $<60$ in two consecutive measurements three months apart. Our definition based on a single measure may lead to overestimation of CKD in people with transient and reversible worsening of renal function, especially in early CKD. Our definition of CKD such as at least stage 3A CKD or higher by eGFR can result in subjects who may be stage 1 and 2 CKD in both the baseline and follow-up being included in the non-CKD population, leading to diluting the association. Our non-significant results for early CKD may be due to this limitation. The other limitation was that we could not use other biomarkers (i.e., the urine albumin to creatinine ratio, etc.) to ascertain new CKD cases since this cohort had only baseline information of biomarkers. Second, in the general population, people with temporary and reversible renal deterioration may limit dietary intake of some nutrients on the recommendation of a doctor, which may lead to reverse causation in our study results. To overcome this limitation to some extent, we additionally analyzed the restricted subjects who were excluded due to being new CKD cases that occurred within 2 years, and the results in the restricted subjects were confirmed to be consistent with those in the total subjects. Third, some dietary intakes in our population were at a very high (e.g., sodium) or very low level (e.g., calcium, potassium, vitamin A, and folate) beyond the recommended dose (RDA or AI). Nutritional deficiency may be due to low socioeconomic status (e.g., income, education levels). Thus, there may be limited generalizability for some results. Fourth, there is no information on dietary intake levels, suggesting the minimum risk level of CKD incidence among the general population to date. Although we considered a certain range at recommended intake levels (RDA or AI) as the minimum risk level, and categorized dietary intake levels according to increasing or decreasing a certain range, it is not clear whether the classification for our reference level is appropriate. Further studies on the appropriate intake levels confirming minimum CKD risk will be needed. However, this study has several strengths. The large sample size provided good statistical power to the results. As our results are for the association between the new cases of CKD development and dietary intake through 12 year follow-up observation of the general population, clinicians may be able to provide specific recommendations for patients based on these findings when planning nutrition management for people at risk of developing CKD. Nonetheless, the association with dietary intake in this study does not mean an association with exacerbation in patients with chronic kidney disease. In particular, it is obvious that high phosphorus intake for CKD patients should be restricted to prevent CKD progression.

\section{Conclusions}

In conclusion, low dietary intakes of phosphorus, vitamin B2, and folate and high dietary intake of vitamins $B 6$ and $C$ were associated with an increased risk of CKD stage $3 \mathrm{~B}$ and over, compared with the intake at recommended levels. There are few studies on the development of new CKD cases in normal subjects and there is sparse experimental and epidemiologic evidence for the association between CKD development and low or 
high dietary factors, including phosphorus intake, to date. Therefore, we could not find a plausible biological mechanism linking low phosphorus and high vitamin B6 intakes to increased CKD risk. Further investigations are required and should accumulate to elucidate the plausible mechanism that links dietary intake to CKD.

Supplementary Materials: The following are available online at https://www.mdpi.com/article/ 10.3390/nu13051517/s1. Table S1: Dietary recommendation and average dietary intake of mineral and vitamin intakes for the general population (KNHENAS 2001) and the Ansan-Ansung cohort study participants (baseline 2001-2002; followed by 2014), a cohort of the KoGES (Korea Genome and Epidemiologic Study. Table S2: Baseline characteristics of study subjects in the 9079 AnsanAnsung KoGES cohort population with 12 year follow-up. Table S3: Single-micronutrient intake and $\mathrm{CKD}^{1}$ risk in the restricted cohort population $(n=8901)$. Table S4: Micronutrients and risk of CKD development $($ GFR $<60)$ in the full multivariable model (clinico-nutritional model) controlling multiple nutrients and additional risk factors. Table S5: Micronutrients and CKD stages in the full multivariable model (clinico-nutritional model) controlling multiple nutrients and additional risk factors. Table S6: Single-micronutrient intake and risk of diabetic and hypertensive CKD stage 3B and over in the entire cohort population $(n=9079)$ and the restricted cohort population $(n=8901)$. Table S7: Micronutrients and risk of diabetic and hypertensive CKD stage 3B and over in the full multivariable model (clinico-nutritional model) controlling multiple nutrients and additional risk factors. Supplementary Figure S1. Receiver operating characteristic (ROC) curves and Harrell's C-index showing the discriminant accuracy of each model for the ability to distinguish new cases of diabetic or hypertensive $\mathrm{CKD}$ stage $3 \mathrm{~B}$ and over in the entire cohort population over 12 year follow-up periods: $[\mathrm{A}]$ diabetic $\mathrm{CKD}$ stage $3 \mathrm{~B}$ and over [B] hypertensive $\mathrm{CKD}$ stage $3 \mathrm{~B}$ and over.

Author Contributions: J.L. analyzed the data, drafted, reviewed, and edited the manuscript, and contributed to the discussion; S.-K.P. conducted, designed, and supervised the study, reviewed and edited the manuscript, and contributed to the discussion; K.-H.O. reviewed and edited the manuscript and contributed to the discussion. All authors have read and agreed to the published version of the manuscript.

Funding: This research was supported by a grant of the Korea Health Technology R\&D Project through the Korea Health Industry Development Institute (KHIDI), funded by the Ministry of Health \& Welfare, Republic of Korea (grant number: HI16C1127).

Institutional Review Board Statement: This study was conducted according to the guidelines of the Declaration of Helsinki, and approved by the Institutional Review of Seoul National University Hospital (protocol code 2104-194-1214 and date of approval 2021/04/29).

Informed Consent Statement: Informed consent was obtained from all subjects involved in this study.

Data Availability Statement: Data described in the manuscript, code book, and analytic code will be made available upon request pending.

Acknowledgments: This study was partially supported by a grant from Seoul National University Hospital (2021). This study was provided with bioresources from National Biobank of Korea, the Centers for Disease Control and Prevention, Republic of Korea (4845-301, 4851-302 and -307).

Conflicts of Interest: The authors declare no conflict of interest.

\section{References}

1. Levey, A.S.; Eckardt, K.-U.; Tsukamoto, Y.; Levin, A.; Coresh, J.; Rossert, J.; Zeeuw, D.D.; Hostetter, T.H.; Lameire, N.; Eknoyan, G. Definition and classification of chronic kidney disease: A position statement from Kidney Disease: Improving Global Outcomes (KDIGO). Kidney Int. 2005, 67, 2089-2100. [CrossRef] [PubMed]

2. Jha, V.; Garcia-Garcia, G.; Iseki, K.; Li, Z.; Naicker, S.; Plattner, B.; Saran, R.; Wang, A.Y.-M.; Yang, C.-W. Chronic kidney disease: Global dimension and perspectives. Lancet 2013, 382, 260-272. [CrossRef]

3. Park, J.I.; Baek, H.; Jung, H.H. Prevalence of chronic kidney disease in Korea: The korean national health and nutritional examination survey 2011-2013. J. Korean Med. Sci. 2016, 31, 915. [CrossRef]

4. Locatelli, F.; Del Vecchio, L.; Pozzoni, P. The importance of early detection of chronic kidney disease. Nephrol. Dial. Transplant. 2002, 17, 2-7. [CrossRef] 
5. Haroun, M.K.; Jaar, B.G.; Hoffman, S.C.; Comstock, G.W.; Klag, M.J.; Coresh, J. Risk Factors for Chronic Kidney Disease: A Prospective Study of 23,534 Men and Women in Washington County, Maryland. J. Am. Soc. Nephrol. 2003, 14, $2934-2941$. [CrossRef] [PubMed]

6. Kazancioğlu, R. Risk factors for chronic kidney disease: An update. Kidney Int. Suppl. 2013, 3, 368-371. [CrossRef] [PubMed]

7. Farhadnejad, H.; Asghari, G.; Mirmiran, P.; Yuzbashian, E.; Azizi, F. Micronutrient Intakes and Incidence of Chronic Kidney Disease in Adults: Tehran Lipid and Glucose Study. Nutrients 2016, 8, 217. [CrossRef]

8. Huang, X.; Jiménez-Moleón, J.J.; Lindholm, B.; Cederholm, T.; Ärnlöv, J.; Risérus, U.; Sjögren, P.; Carrero, J.J. Mediterranean Diet, Kidney Function, and Mortality in Men with CKD. Clin. J. Am. Soc. Nephrol. 2013, 8, 1548-1555. [CrossRef]

9. Lin, J.; Fung, T.T.; Hu, F.B.; Curhan, G.C. Association of Dietary Patterns with Albuminuria and Kidney Function Decline in Older White Women: A Subgroup Analysis from the Nurses' Health Study. Am. J. Kidney Dis. 2011, 57, 245-254. [CrossRef]

10. Kim, J.; Lee, J.; Kim, K.-N.; Oh, K.-H.; Ahn, C.; Lee, J.; Kang, D.; Park, S.K. Association between Dietary Mineral Intake and Chronic Kidney Disease: The Health Examinees (HEXA) Study. Int. J. Environ. Res. Public Health 2018, 15, 1070. [CrossRef]

11. Kim, Y.; Han, B.-G.; Group, K. Cohort profile: The Korean genome and epidemiology study (KoGES) consortium. Int. J. Epidemiol. 2017, 46, e20. [CrossRef] [PubMed]

12. Levey, A.S.; Coresh, J.; Balk, E.; Kausz, A.T.; Levin, A.; Steffes, M.W.; Hogg, R.J.; Perrone, R.D.; Lau, J.; Eknoyan, G. National Kidney Foundation Practice Guidelines for Chronic Kidney Disease: Evaluation, Classification, and Stratification. Ann. Intern. Med. 2003, 139, 137-147. [CrossRef] [PubMed]

13. Levey, A.S.; Stevens, L.A.; Schmid, C.H.; Zhang, Y.; Castro, A.F., III; Feldman, H.I.; Kusek, J.W.; Eggers, P.; Van Lente, F.; Greene, T. A new equation to estimate glomerular filtration rate. Ann. Int. Med. 2009, 150, 604-612. [CrossRef]

14. Abutaleb, N. Why we should sub-divide CKD stage 3 into early (3a) and late (3b) components. Nephrol. Dial. Transplant. 2007, 22, 2728-2729. [CrossRef] [PubMed]

15. Zahran, A.; Shoker, A. About CKD stage-3 subdivision proposal. Nephrol. Dial. Trans. 2008, 23, 1765. [CrossRef]

16. Yang, J.J.; Yang, J.H.; Kim, J.; Cho, L.Y.; Park, B.; Ma, S.H.; Song, S.H.; Min, W.-K.; Kim, S.S.; Park, M.S.; et al. Reliability of Quadruplicated Serological Parameters in the Korean Genome and Epidemiology Study. Epidemiol. Health 2011, 33, e2011004. [CrossRef]

17. Bellasi, A.; Mandreoli, M.; Baldrati, L.; Corradini, M.; Di Nicolò, P.; Malmusi, G.; Santoro, A. Chronic Kidney Disease Progression and Outcome According to Serum Phosphorus in Mild-to-Moderate Kidney Dysfunction. Clin. J. Am. Soc. Nephrol. 2011, 6, 883-891. [CrossRef]

18. Chang, A.R.; Miller, E.R., III; Anderson, C.A.; Juraschek, S.P.; Moser, M.; White, K.; Henry, B.; Krekel, C.; Oh, S.; Charleston, J. Phosphorus additives and albuminuria in early stages of CKD: A randomized controlled trial. Am. J. Kidney Dis. 2017, 69, 200-209. [CrossRef]

19. Calo, L.A.; Savica, V.; Davis, P.A. Phosphate Content of Beverages in Addition to Food Phosphate Additives: Real and Insidious Danger for Renal Patients. J. Ren. Nutr. 2012, 22, 292-293. [CrossRef] [PubMed]

20. Chang, A.R.; Lazo, M.; Appel, L.J.; Gutiérrez, O.M.; Grams, M.E. High dietary phosphorus intake is associated with all-cause mortality: Results from NHANES III. Am. J. Clin. Nutr. 2013, 99, 320-327. [CrossRef] [PubMed]

21. Zhu, Y.; Liu, X.; Li, N.; Cui, L.; Zhang, X.; Liu, X.; Yu, K.; Chen, Y.; Wan, Z.; Yu, Z. Association Between Iron Status and Risk of Chronic Kidney Disease in Chinese Adults. Front. Med. 2020, 6, 303. [CrossRef] [PubMed]

22. Nurko, S.; Weigelt, J.A. Anemia in chronic kidney disease: Causes, diagnosis, treatment. Clevel. Clin. J. Med. 2006, 73, 289-297. [CrossRef]

23. Martines, A.M.F.; Masereeuw, R.; Tjalsma, H.; Hoenderop, J.G.; Wetzels, J.F.M.; Swinkels, D.W. Iron metabolism in the pathogenesis of iron-induced kidney injury. Nat. Rev. Nephrol. 2013, 9, 385-398. [CrossRef] [PubMed]

24. Nakanishi, T.; Kuragano, T.; Nanami, M.; Hasuike, Y. Iron Localization and Infectious Disease in Chronic Kidney Disease Patients. Am. J. Nephrol. 2016, 43, 237-244. [CrossRef]

25. Gibson, R. Assessment of the status of thiamin, riboflavin and niacin. In Principles of Nutritional Assessment; Oxford University Press: Oxford, UK, 2005; pp. 545-574.

26. Skoupy, S.; Födinger, M.; Veitl, M.; Perschl, A.; Puttinger, H.; Röhrer, C.; Schindler, K.; Vychytil, A.; Hörl, W.H.; Sunder-Plassmann, G. Riboflavin Is a Determinant of Total Homocysteine Plasma Concentrations in End-Stage Renal Disease Patients. J. Am. Soc. Nephrol. 2002, 13, 1331-1337. [CrossRef] [PubMed]

27. Xie, D.; Yuan, Y.; Guo, J.; Yang, S.; Xu, X.; Wang, Q.; Li, Y.; Qin, X.; Tang, G.; Huo, Y.; et al. Hyperhomocysteinemia predicts renal function decline: A prospective study in hypertensive adults. Sci. Rep. 2015, 5, 16268. [CrossRef] [PubMed]

28. Ferraro, P.M.; Taylor, E.N.; Gambaro, G.; Curhan, G.C. Vitamin B6 intake and the risk of incident kidney stones. Urolithiasis 2017, 46, 265-270. [CrossRef]

29. Lindner, A.; Bankson, D.D.; Stehman-Breen, C.; Mahuren, J.; Coburn, S.P. Vitamin B6 metabolism and homocysteine in end-stage renal disease and chronic renal insufficiency. Am. J. Kidney Dis. 2002, 39, 134-145. [CrossRef]

30. House, A.A.; Eliasziw, M.; Cattran, D.C.; Churchill, D.N.; Oliver, M.J.; Fine, A.; Dresser, G.K.; Spence, J.D. Effect of B-vitamin therapy on progression of diabetic nephropathy: A randomized controlled trial. JAMA 2010, 303, 1603-1609. [CrossRef] [PubMed]

31. Xu, X.; Qin, X.; Li, Y.; Sun, D.; Wang, J.; Liang, M.; Wang, B.; Huo, Y.; Hou, F.F. Efficacy of folic acid therapy on the progression of chronic kidney disease: The renal substudy of the China Stroke Primary Prevention Trial. JAMA Intern. Med. 2016, 176, 1443-1450. [CrossRef] [PubMed] 
32. Ferraro, P.M.; Curhan, G.C.; Gambaro, G.; Taylor, E.N. Total, Dietary, and Supplemental Vitamin C Intake and Risk of Incident Kidney Stones. Am. J. Kidney Dis. 2016, 67, 400-407. [CrossRef] [PubMed]

33. Lamarche, J.; Nair, R.; Peguero, A.; Courville, C. Vitamin C-Induced Oxalate Nephropathy. Int. J. Nephrol. 2011, $2011,146927$. [CrossRef]

34. Middleton, J.P.; Lehrich, R.W. Prescriptions for dietary sodium in patients with chronic kidney disease: How will this shake out? Kidney Int. 2014, 86, 457-459. [CrossRef] [PubMed]

35. Lin, J.; Hu, F.B.; Curhan, G.C. Associations of Diet with Albuminuria and Kidney Function Decline. Clin. J. Am. Soc. Nephrol. 2010, 5, 836-843. [CrossRef]

36. Kondaiah, P.; Yaduvanshi, P.S.; Sharp, P.A.; Pullakhandam, R. Iron and Zinc Homeostasis and Interactions: Does Enteric Zinc Excretion Cross-Talk with Intestinal Iron Absorption? Nutrients 2019, 11, 1885. [CrossRef] 